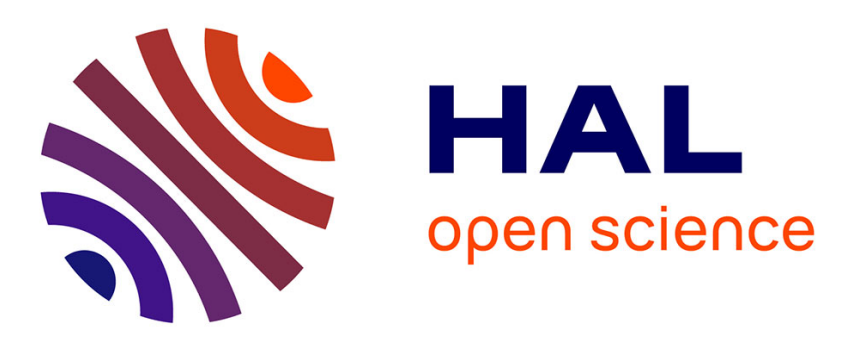

\title{
Distributed Algorithm for Egalitarian Matching between Individuals and Activities with Additively Separable Preferences
}

Maxime Morge, Antoine Nongaillard

\section{- To cite this version:}

Maxime Morge, Antoine Nongaillard. Distributed Algorithm for Egalitarian Matching between Individuals and Activities with Additively Separable Preferences. 2017 IEEE 29th International Conference on Tools with Artificial Intelligence (ICTAI), Nov 2017, Boston, France. 10.1109/ICTAI.2017.00117 . hal-01811517

\section{HAL Id: hal-01811517 https://hal.science/hal-01811517}

Submitted on 5 Nov 2020

HAL is a multi-disciplinary open access archive for the deposit and dissemination of scientific research documents, whether they are published or not. The documents may come from teaching and research institutions in France or abroad, or from public or private research centers.
L'archive ouverte pluridisciplinaire HAL, est destinée au dépôt et à la diffusion de documents scientifiques de niveau recherche, publiés ou non, émanant des établissements d'enseignement et de recherche français ou étrangers, des laboratoires publics ou privés. 


\section{Distributed Algorithm for Egalitarian Matching between Individuals and Activities with Additively Separable Preferences}

\author{
Maxime Morge \\ Univ. Lille, CNRS, Centrale Lille \\ UMR 9189 - CRIStAL \\ F-59000 Lille, France \\ Email: Maxime.Morge@univ-lille.fr
}

\author{
Antoine Nongaillard \\ Univ. Lille, CNRS, Centrale Lille \\ UMR 9189 - CRIStAL \\ F-59000 Lille, France \\ Email: Antoine.Nongaillard@univ-lille.fr
}

\begin{abstract}
We aim at providing a social network such that users form groups to practice together some activities. In this paper, we present a formal framework for coalition formation which is suitable for our usecase. We restrict ourselves to additively separable preferences whose representation is linear with respect to the inputs. We propose here a distributed algorithm which aims at maximizing the egalitarian welfare of the resulting matching. Our experiments shows that: i) this algorithm reaches a better outcome than the classical local search techniques; and ii) the distribution of our algorithm speeds up its runtime.
\end{abstract}

\section{INTRODUCTION}

Multiagent systems (MAS) is a relevant paradigm for the analysis, the design and the implementation of systems composed of autonomous entities in interaction. In order to design socio-technical systems for mediation or simulation, MAS allow to model the feedback loops between heterogeneous actors whose local decision-making leads to complex global phenomena. In this perspective, one of the challenges of the MAS community is to facilitate the elicitation of preferences.

Our work is part of a project which aims at understanding and modelling the dynamic of feedback occurring in a group of individuals interacting both within a virtual and a real social network. The usecase on which we are focusing in this project consists of forming some groups of seniors (60-70 years old) in order to share some activities. This scenario involves several thousand users. We aim at maximizing the satisfaction of users in order to improve social cohesion and avoid the isolation of seniors. We want to propose a social network such that the users form groups to practice together some activities. The purpose of this system is to suggest to each user some peers with whom practicing these activities.

In this paper, we present a formal framework for coalition formation which is suitable for our usecase. A set of individuals must be matched together in order to share one of the activities with respect to the preferences of individuals for their peers and for the activities. We restrict ourselves to additively separable preferences, whose representation is linear with respect to the number of individuals, in order to propose an algorithm which returns a "good" matching where no activity is oversubscribed. We propose here an algorithm which aims at maximizing the egalitarian welfare, i.e. the satisfaction of the worst-off individual. By adopting the model of actor [1], we are able to distribute this algorithm. Our experiments show that the solution of our algorithm has a better quality than those obtained with the classical local search techniques. Moreover, its distribution speeds up its runtime (up to 5 times).

Section II compares our approach with the related works. We introduce our matching problem in Section III. We propose a matching algorithm in Section IV. The distribution of this algorithm consists of agent behaviours described in Section V. Section VI exhibits our empirical results. Finally, we conclude with some directions for future work (cf Section VII).

\section{RELATED WORKS}

Social choice theory aims at designing and analyzing collective decision-making processes which imply a set of agents selecting or classifying a subset of alternatives among the available ones. Contrary to Economy, Computer science is concerned in this field by the study of algorithms in order to propose operational methods. We focus here on a particular matching problem.

In the problem of hedonic coalition formation, which has been formalized in [2], each player is endowed with a single preference relation over all the coalitions which contain this player. Our problem is a specialization of the hedonic coalition problem. We can represent our problem as a hedonic game. For this purpose, we associate : i) one player for each activity such that all the coalitions which are compliant with its capacity are equally preferred; and ii) one player for each individual whose preferences are deduced from the utility function of the latter. Even if the resulting preferences are not additively separable as in [3], we can represent them using rational lists for coalitions (RLC) [4]. To our best knowledge, there exists no algorithm which computes a partition maximizing the "egalitarian" welfare. Indeed, we consider here that the preferences over the activities and the preferences over the individuals are independent, then we aggregate them in an utility function. Under this assumption, our setting has the useful 
structural properties of two-sided matching that distinguish it from a generic hedonic game. The fact that the activities play the role of focal points [5] and the succinct representation of individual's preferences allow us to propose some algorithms to reach "good" matchings.

The problem of group activity selection has been proposed in [6]. In such a problem, each player participates in at most one activity and its preferences over activities depend on the number of participants in the activity. This is a generalization of anonymous hedonic games. Even if this problem has been extended [7] in order to take into account the relationships among the agents, the latter are encoded by a social network, i.e. an undirected graph where nodes correspond to agents and edges represent communication links between them. By contrast, in our problem, each individual is endowed with a preference relation over the activities and a preference relation over its peers.

The Hospital/Resident $(H R)$ problem has been introduced in [8]. This problem is a specialization of the coalition formation game where a set of residents must be assigned to the hospitals in accordance with the preferences of the residents over the hospitals and the preferences of hospitals over the residents. The HR problem has many extensions [9]. To the best of our knowledge, none extension is suitable for our usecase.

How does an agent evaluate its preferences? We adopt here utility functions (cardinal preferences). In order to reduce the users' effort, we assume some additively separable preferences and that the evaluation of activities and individuals are comparable. Even if its expressiveness is limited, the representation of our preferences is linear with respect to the number of individuals.

What is the "best" solution for collective decision-making problem? In the literature, two kinds of rules derive the social choice from the individual preferences: the first ones are based on the desirable properties of the solution (e.g. stability or Pareto-optimality), while the latter are based on the aggregation of the individual preferences (e.g. the utilitarian/egalitarina welfare). We adopt the second approach since none concept from the first approach is suitable. In particular, we propose here an algorithm which aims at maximizing the egalitarian welfare. For this purpose, we are considering a decision criterion for the "activity" agent which is evaluated only if the activity is full.

How to reach a matching which maximizes the utilitarian/egalitarian welfare? This problem can be NP-hard. For this purpose, we can consider Distributed Constraint Optimization Problem (DCOP) algorithm or Local Search Techniques (LST). It has been shown in [10] that DCOP algorithms are not necessarily scalable for matching problems. However, LST are not suitable when the function to be optimized consists of many local optima. That is the reason why we adopt a multiagent approach, in particular a multi-level model as recommended by [11] where each "activity" agent represents a group of individuals.

\section{IA PROBLEM}

We abstract away from our practical application to introduce a formal framework for coalition formation which captures our usecase.

We present here the individuals/activities (IA) problem. In such a problem, the individuals selects their favorite activities with the partners they prefer.

Definition 1 (IA Problem): An individuals/activities (IA) problem of size $(m, n)$, with $m \geq 1$ and $n \geq 1$, is a couple $I A=\langle I, A\rangle$ with $m$ individuals and $n$ activities, where:

- $A=\left\{a_{1}, \ldots, a_{n}\right\}$ is a set of $n$ activities. Each activity $a_{j}$ has a positive integral capacity $c_{j}$

- $I=\{1, \ldots, m\}$ is a set of $m$ individuals. Each individual $i$ is endowed with,

1) a preference relation over the activities, i.e. a reflexive, complete and transitive preference ordering $\unrhd_{i}$ over the activities $A \cup\{\theta\}$, including the void activity (denoted $\theta$ ). The corresponding strict preference relation is denoted by $\triangleright_{i}$,

2) a purely hedonic preference, i.e. a reflexive, complete and transitive preference ordering $\succsim_{i}$ over the set of groups it belongs $G(i)=\{G \subseteq I ; i \in G\}$. The corresponding strict preference relation is denoted by $\succ_{i}$.

Intuitively, the void activity corresponds to do nothing.

We aim at forming coalitions of individuals around the activities.

Definition 2 (Coalition): Let $I A=\langle I, A\rangle$ be an IA problem. A coalition is a couple $C=\langle a, G\rangle$ where $a \in A \cup\{\theta\}$ and $G \subseteq I$. The activity of a coalition $C$ is $a_{C}$ with a capacity ${ }^{1}$ $c_{C}$ and the group $G_{C}$. A non-empty coalition $C$ is such that $G_{C} \neq \emptyset$ and $C$ is for $i$ if $i \in G_{C}$.

We expect the number of individuals to be considerably larger than the number of available activities $(m>>n)$.

Definition 3 (Matching): A matching $M$ for the problem $I A=\langle I, A\rangle$ is represented by the functions $a_{M}: I \rightarrow A \cup\{\theta\}$ and $g_{M}: I \rightarrow \mathcal{P}(I)$ such that:

$$
\begin{array}{r}
\forall i \in I, a_{M}(i) \in A \cup\{\theta\} \\
\forall i \in I, i \in g_{M}(i) \subseteq I \\
\forall i \in I, a_{M}(i)=\theta \Rightarrow g_{M}(i)=\{i\} \\
\forall i \in I \forall j \in g_{M}(i), a_{M}(j)=a_{M}(i) \\
\forall i, j \in I, i \neq j \wedge a_{M}(i)=a_{M}(j) \neq \theta \Rightarrow \\
g_{M}(i)=g_{M}(j)
\end{array}
$$

The assignment of an individual is an activity, possibly the void activity (cf equation 1). In this case, the individual is said inactive. Each individual is associated with the group it belongs (cf equation 2). All the individuals which are assigned to the void activity are alone (cf equation 3 ). All the individuals which are associated with each others have the same activity (cf equation 4) and reciprocally all the individuals which are assigned to the same activity, excepted the void activity, are associated with each other (cf equation 5).

\footnotetext{
${ }^{1}$ the void activity has an infinite capacity.
} 
In order to simplify the notations, we introduce the post function of a matching $M$ which returns the set of individuals assigned to each activity:

$$
\begin{aligned}
p_{M}: A \cup\{\theta\} & \rightarrow \mathcal{P}(i) \\
p_{M}(a) & =\left\{i \in I ; a_{M}(i)=a\right\}
\end{aligned}
$$

The posts of an activity can be empty. If $a_{M}(i)=\theta$, we say that $i$ is not assigned. An activity $a \in A$ is:

- oversubscribed if $\left|p_{M}(a)\right|>c_{a}$;

- full if $\left|p_{M}(a)\right|=c_{a}$;

- undersubscribed if $\left|p_{M}(a)\right|<c_{a}$.

A matching is said sound if no activity is oversubscribed.

A matching is a coalition structure, i.e. a partition of individuals.

Property 1 (Partition): Let $M$ be a matching for the problem $I A=\langle I, A\rangle$. We verify:

$$
\forall a \in A \cup\{\theta\},\left\langle a, p_{M}(a)\right\rangle \text { is a coalition }
$$

$$
\cup_{a \in A \cup\{\theta\}} p_{M}(a)=I \wedge \forall a_{i}, a_{j} \in A \cup\{\theta\} p_{M}\left(a_{i}\right) \cap p_{M}\left(a_{j}\right)=\emptyset
$$

Proof 1: Straight forward from Def. 2 and Def. 3.

The coalition which contains $i$ in the matching $M$ is denoted $C_{M}(i)$.

Each individual evaluates its preferences over the coalitions and so the matchings regarding the group it belongs and the activity it is assigned.

Definition 4 (Preferences): Let $I A=\langle I, A\rangle$ be an IA problem.

- Let $C$ and $C^{\prime}$ be two coalitions for $i$.

- The individual $i$ prefers $C$ to $C^{\prime}\left(\right.$ denoted $\left.C \succsim_{i} C^{\prime}\right)$ iff:

$$
a_{C} \unrhd_{i} a_{C^{\prime}} \wedge G_{C} \succsim_{i} G_{C^{\prime}}
$$

- The individual $i$ strictly prefers $C$ to $C^{\prime}$ (denoted $C \succ_{i} C^{\prime}$ ) iff:

$$
C \succsim_{i} C^{\prime} \wedge\left(a_{C} \triangleright_{i} a_{C^{\prime}} \vee G_{C} \succ_{i} G_{C^{\prime}}\right)
$$

- Let $M$ and $M^{\prime}$ be two sound matchings. The individual $i$ prefers $M$ to $M^{\prime}$ (denoted $M \succsim_{i} M$ ) iff:

$$
C_{M}(i) \succsim_{i} C_{M^{\prime}}(i)
$$

The strict preference relation over the matchings is denoted by $\succ_{i}$. An individual prefers a coalition to a second one if it prefers the activity and the group of the first one (cf equation 9). It prefers a matching to a second one if it prefers its coalition in the first one (cf equation 11). The preference relations over the sound matchings are reflexive, transitive and possibly partial.

We now define the desirable properties for a matching.

Definition 5 (Stability): Let $M$ be a matching for the problem $I A=\langle I, A\rangle$.

- $M$ is individually rational (IR) iff:

$$
\forall i \in I,\left(a_{M}(i) \unrhd_{i} \theta\right) \wedge g_{M}(i) \succsim_{i}\{i\}
$$

- A non-empty coalition $C$ strongly blocks $M$ iff:

$$
\begin{gathered}
\left|G_{C}\right| \leq c_{C} \\
\forall i \in G_{C}, C \succ_{i} C_{M}(i)
\end{gathered}
$$

$M$ is core stable (CS) if it is sound and there is no strongly blocking coalition.

- A non-empty coalition $C$ weakly blocks $M$ iff:

$$
\begin{gathered}
\left|G_{C}\right| \leq c_{C} \\
\forall i \in G_{C}, C \succsim_{i} C_{M}(i) \\
\exists i \in G_{C} C \succ_{i} C_{M}(i)
\end{gathered}
$$

$M$ is strict core stable (SCS) if it is sound and there is no weakly blocking coalition.

- $M$ is Nash stable (NS) if it is sound, rational and:

$$
\begin{aligned}
& \forall i \in I \forall a \in A, a \neq a_{M}(i) \text { with } p_{M}(a)<c_{a} \\
& C_{M}(i) \succsim_{i}\left\langle a, p_{M}(a) \cup\{i\}\right\rangle
\end{aligned}
$$

- The matching $M$ is individually stable (IS) if it is sound, rational and:

$$
\begin{aligned}
& \forall i \in I \exists a \in A, a \neq a_{M}(i) \text { with } p_{M}(a)<c_{a} \\
& \left\langle a, p_{M}(a) \cup\{i\}\right\rangle \succ_{i} C_{M}(i) \Rightarrow \\
& \exists j \in p_{M}(a) C_{M}(j) \succ_{j}\left\langle a, p_{M}(a) \cup\{i\}\right\rangle
\end{aligned}
$$

- The matching $M$ is contractually individually stable (CIS) if it is sound, rational and:

$$
\begin{aligned}
& \forall i \in I \exists a \in A, a \neq a_{M}(i) \text { with } p_{M}(a)<c_{a} \\
& \left\langle a, p_{M}(a) \cup\{i\}\right\rangle \succ_{i} C_{M}(i) \Rightarrow \\
& \left(\exists j \in p_{M}(a) C_{M}(j) \succ_{j}\left\langle a, p_{M}(a) \cup\{i\}\right\rangle \vee\right. \\
& \left.\exists j^{\prime} \in g_{M}(i) j^{\prime} \neq i, C_{M}(i) \succ_{j^{\prime}}\left\langle a_{M}(i), g_{M}(i) \backslash\{i\}\right\rangle\right)
\end{aligned}
$$

- The matching $M$ is contractually strict core (CSC) if any weakly blocking coalition $C$ makes at least one individual $j \in I \backslash G_{C}$ worse off when breaking off.

An individual chooses a coalition and so a matching such that it prefers being with its partners to be alone (cf equation 12). Intuitively, the individuals in a blocking coalition would like to separate and form their own coalition, which makes the underlying matching unstable. A coalition strongly blocks a matching if its activity is not oversubscribed (cf equation 13) and all the individuals of the coalition strictly prefer the latter to be assigned according to the matching (cf equation 14). A coalition weakly blocks a matching if its activity is not oversubscribed (cf equation 15), all the individuals of the coalition prefer the latter to be assigned according to the matching (cf equation 16) and at least one individual of the coalition strictly prefers the latter to be assigned according to the matching (cf equation 17). A Nash stable matching is immune to individual movements since the activities are full or the coalition for any individual in the matching is at least as good as any coalition they can join (cf equation 18). A matching is individually stable if no individual can benefit by moving from its coalition to another undersubscribed activity while not making the posts of that activity worse off (cf equation 19). A matching is contractually individually stable if no individually can benefit by moving from its coalition to another activity while making no posts of either activity worse off (cf equation 20).

We now formulate the other desirable properties which are not based on the interpersonal comparisons.

Definition 6 (Pareto-dominanceloptimal): Let $M$ and $M^{\prime}$ be two sound matchings for the problem $I A=\langle I, A\rangle$. 
- $M$ is perfect iff:

$$
\begin{aligned}
& \forall i \in I \forall a \in A \cup\{\theta\} \forall g \in G(i) \\
& \text { it is not the case }\langle a, g\rangle \succ_{i} C_{M}(i)
\end{aligned}
$$

- $M^{\prime}$ Pareto-dominates $M$ iff:

$$
\begin{aligned}
& \forall i \in I, C_{M^{\prime}}(i) \succsim_{i} C_{M}(i) \\
& \exists i \in I, C_{M^{\prime}}(i) \succ_{i} C_{M}(i)
\end{aligned}
$$

A matching is Pareto-optimal (PO) if it is not Paretodominated.

A matching is perfect if the coalition for any individual is a most preferred one for it. A matching Pareto-dominates a second one if it is strictly better for at least one individual and not worst for the others. A matching is Pareto-optimal if there is no alternative in which all agents would be in an equivalent or better position.

As illustrated by the following example, even if the individual rationality of a matching is necessary condition to be either core stable or Nash stable, neither type of stability implies the other. Moreover, an IA problem does not necessarily have a strict core stable matching or a Nash stable one.

Example 1 (Stability): Let us consider the IA problem with 3 individuals $(1,2$ and 3$)$. and one activity $a$ such that $a \unrhd_{i} \theta$ with $i \in\{1,2,3\}$.

Let us first suppose that the capacity of $a$ is 2 and the social preferences are circular:

- $\{1,2\} \succ_{1}\{1\} \succ_{1}\{1,3\}$;

- $\{2,3\} \succ_{2}\{2\} \succ_{2}\{1,2\}$

- $\{1,3\} \succ_{3}\{3\} \succ_{3}\{2,3\}$.

This instance has no core stable matching (and so no strict core stable matching) since the matching $M_{1}$ (with $p_{M_{1}}(a)=$ $\{1,2\})$ is weakly/strongly blocked by the coalition $\langle a,\{2\}\rangle$, the matching $M_{2}$ (with $p_{M_{2}}(a)=\{2,3\}$ ) is weakly/strongly blocked by $\langle a,\{3\}\rangle$ and the matching $M_{3}$ (with $p_{M_{3}}(a)=$ $\{1,3\}$ ) is weakly/strongly blocked by $\langle a,\{1\}\rangle$. Moreover, there is no Nash stable matching. Indeed, $M_{1}, M_{2}$ and $M_{3}$ are not individually rational.

Let us now assume that the capacity of $a$ is $3, a \unrhd_{i} \theta$ and $\theta \unrhd_{i} a$ with $i \in\{1,2,3\}$. We consider that the individual \# 3 is "undesired", i.e. the coalitions with the individual \# 3 are not individually rational for the others:

- $\{1,2\} \succ_{1}\{1\} \succ_{1}\{1,2,3\} \succ_{1}\{1,3\}$;

- $\{1,2\} \succ_{2}\{2\} \succ_{2}\{1,2,3\} \succ_{2}\{2,3\}$;

- $\{2,3\} \succ_{3}\{1,2,3\} \succ_{3}\{1,3\} \succ_{3}\{3\}$;

The matching $M_{1}$ such that $p_{M_{1}}(a)=\{1,2\}$ is strict core stable and so: i) core stable and individual rational; and ii) Paretooptimal and contractually individually stable. Moreover, it is not Nash stable. The matching $M_{2}$ such that $p_{M_{2}}(a)=\{3\}$ is Nash stable and so (contractually) individually stable but it is not core stable (and so neither strict core stable). The matching $M_{3}$ such that $p_{M 3}(a)=\{1,2,3\}$ is Pareto-optimal and so contractually individually stable but it is not individually rational.

Even if the strict core stability and the Nash stability are desirable property, there exists no necessarily such solutions.
By contrast, the Pareto-optimality, and so the contractually individually stability, seems not to be discriminative. An alternative way to assess the quality of a matching consists of the concept of social welfare. For this purpose, we assume that the individuals have cardinal preferences. Moreover, in an IA problem, each individual evaluates its preferences with respect to $2^{m-1}$ groups. This preference representation takes exponential space. By contrast, the representation of additively separable preferences is linear with respect to the number of individuals.

Definition 7 (Additively separable IA): Let $I A=\langle I, A\rangle$ be a IA problem of size $(m, n)$. The problem is additively separable (ASIA) if each individual $i \in I$ is endowed with:

1) a valuation function $v_{i}: A \cup\{\theta\} \rightarrow[-1 ; 1]$ representing its preferences over the activities, possibly the void activity such as $v_{i}(\theta)=0$;

2) a valuation function $w_{i}: I \backslash\{i\} \rightarrow[-1 ; 1]$ representing its preferences over the potential partners.

The utility for an individual $i$ is the function $u_{i}: G(i) \times A \cup$ $\{\theta\} \rightarrow[-1 ; 1]$ defined such that:

$\forall g \in G(i) \forall a \in A \cup\{\theta\}, u_{i}(g, a)=\frac{\left[\frac{1}{m-1} \Sigma_{j \in g, j \neq i} w_{i}(j)\right]+v_{i}(a)}{2}$

We assumes that the preferences over the individuals and the preferences over the activities are comparable. In particular, the utility for an individual to be alone only depends on its valuation of the activity. Moreover, the satisfaction of an individual with respect to a group is the mean valuation of its partners. In the rest of the paper, we do not consider that the utilities are defined to correspond to ordinal preferences as in [12] but we suppose a direct access the utilities of agents since it is the case in our practical application.

Contrary to the Paretian approach, the social choice theory analyses the interpersonal comparisons [13]. This theory reconciles the utilitarianism of Jeremy Bentham and the distributive justice advocated by John Rawls [14].

Definition 8 (Social welfare): Let $I A=\langle I, A\rangle$ be an ASIA problem of size $(m, n) M$

- The utilitarian welfare of a matching $M$ is:

$$
U(M)=\frac{1}{m} \sum_{i \in I} u_{i}\left(g_{M}(i), a_{M}(i)\right)
$$

- The egalitarian welfare of a matching $M$ is:

$$
E(M)=\min _{i \in I}\left(u_{i}\left(g_{M}(i), a_{M}(i)\right)\right)
$$

The higher the welfare is, the better the matching is. A maximum utilitarian (resp. egalitarian) matching maximizes the utilitarian (resp. egalitarian) welfare. It is worth noticing that the egalitarian welfare is 0 if at least one individual is inactive.

Inspired by [3], Figure 1 depicts the inclusions relationships between concepts which follow from their definitions.

Example 2 (ASIA problem): Let us consider our previous example where the capacity of the activity is 3 and individual \# 3 is undesired. We define the valuation functions such that $v_{1}(a)=v_{2}(a)=v_{3}(a)=0, w_{1}(2)=w_{2}(1)=\frac{1}{2}$, 


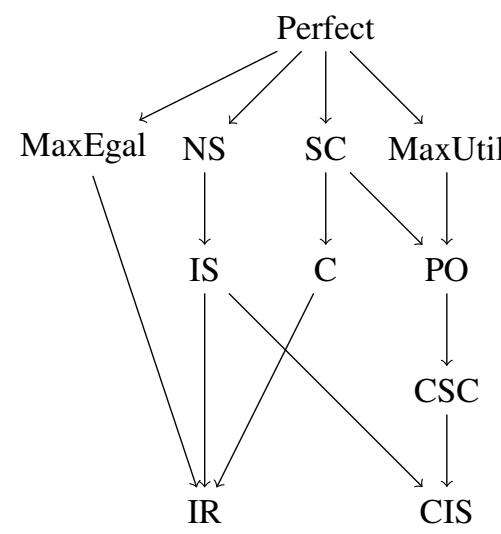

Fig. 1: Inclusion relationships between stability, fairness and optimality for IA problems. For instance, each strict core matching is Pareto-optimal

$w_{1}(3)=w_{2}(3)=-1, w_{3}(1)=\frac{1}{2}$ and $w_{3}(2)=1$. Therefore, the induced ordinal preferences are as previously. While the matching $M_{1}$ (such that $p_{M_{1}}(a)=\{1,2\}$ ) maximizes the utilitarian welfare $\left(U\left(M_{1}\right)=\frac{1}{12}\right)$ and the egalitarian welfare $\left(E\left(M_{1}\right)=0\right)$, it is not the case of the matching $M_{3}$ (such that $p_{M 3}(a)=\{1,2,3\}$ ) since its utilitarian welfare $\left(U\left(M_{3}\right)=\frac{1}{24}\right)$ and it egalitarian welfare $\left(E\left(M_{3}\right)=-\frac{1}{8}\right)$ are smaller.

\section{THE ALGORITHM}

We propose here an algorithm which aims at maximizing the egalitarian welfare of the resulting matching for any ASIA problem instance.

In Algorithm 1, all the individuals are initially alone and assigned to the void activity. Each in turn, a free individual $i$ considers the rational activity $a$ it prefers. The algorithm is inclusive: if $a$ is not full, then $i$ is assigned. Otherwise, the algorithm tries to improve the egalitarian welfare of the group, eventually by firing the individuals whose presence penalizes the egalitarian welfare of the group. If the assignment of $i$ does not improve the egalitarian welfare of the group then $i$ must concede, i.e. consider the next rational activity. The individuals which are replaced by $i$ must concede. An agent which is rejected by all its rational activities stay alone and it is definitely assigned to the void activity. An approximation algorithm consists of excluding only one individual at each step (line 20).

It can be noticed that our (approximation) algorithm always returns a sound matching.

Property 2 (Termination): Our (approximation) algorithm applied over an ASIA problem ends and returns a sound matching.

Proof 2 (Termination): Let $I A=\langle I, A\rangle$ be an ASIA problem. We consider the following loop invariant:

$$
\Sigma_{i \in I} \mid \text { concessions }(i)|+| \text { Free } \mid
$$

This invariant is positive. It strictly decreases after each loop since:

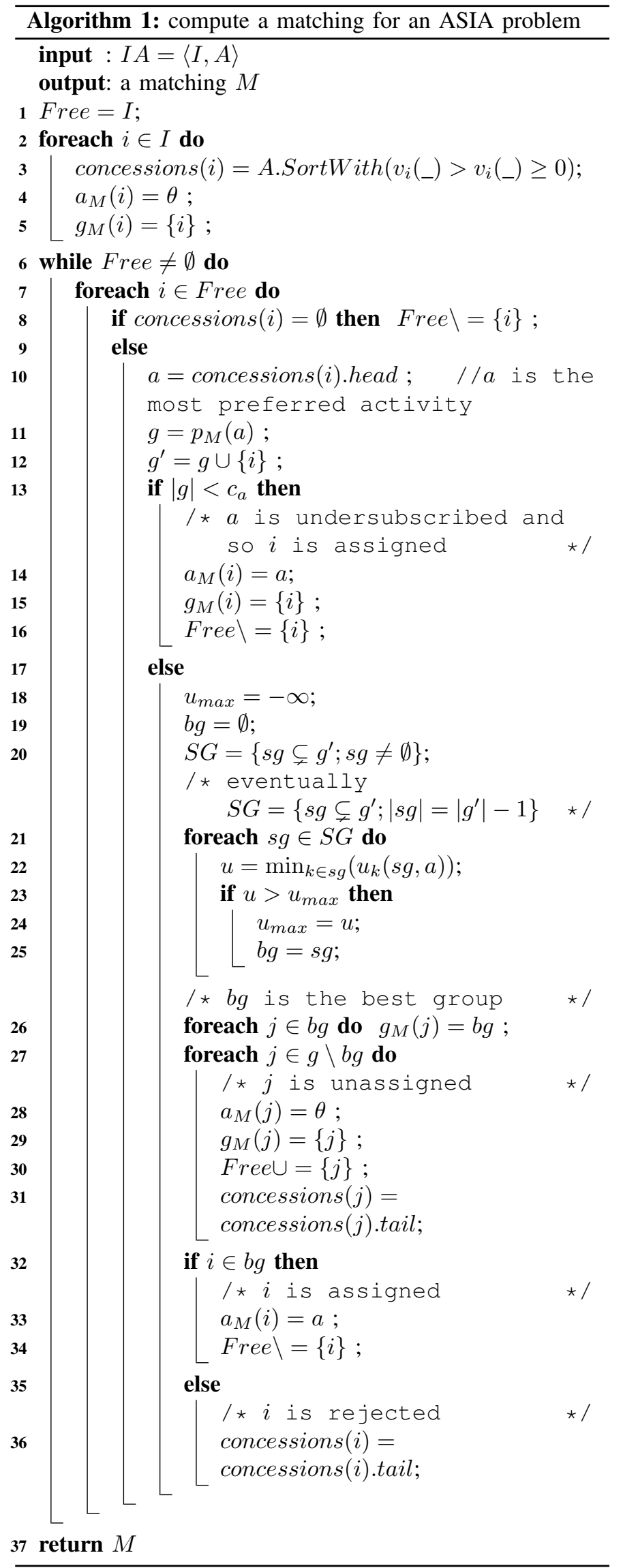


1) an individual, which is assigned, is removed from Free;

2) an individual, which is not assigned, concedes until it is definitely assigned to the void activity;

3) any individual, which is unassigned, concedes and at least one another individual is assigned (and so removed from Free).

The resulting matching is sound since the activities are never oversubscribed.

It is worth noticing that our algorithm does not always maximize the egalitarian welfare since the heuristic gives priority to the activities.

Example 3 (ASIA problem): Let us consider our previous example (cf Example 2). The matching $M_{3}$ such that $p_{M 3}(a)=\{1,2,3\}$ is reached by our algorithm even it is not individually rational.

\section{Agent BeHAVIOURS}

We consider the asynchronous message-passing model of actor for concurrent programming [1]. In this perspective, the primitives are agents and events. An agent represents an independent program that runs on its own processor. An event is the creation of an agent or the utterance/reception of a message. It is worth noticing that the system is distributed since the message transmission delay is arbitrary but not negligible. The underlying channels are assumed to be reliable (a message is delivered once and only once) and that the messages may arrive in different order from sending.

In order to propose a distributed solver based on this model, we distinguish 3 kinds of agents:

1) the "solver" agent which creates the other agents and records the assignments;

2) the "individual" agents which are endowed with the same behaviours but with different preferences;

3) the "activity" agents which are endowed with the same behaviours but managing different groups and capacities.

The behaviour of the "solver" agent consists of: i) creating other agents; ii) triggering the solving; iii) recording the assignments/unassignments; and iv) returning the matching when all the individual are assigned.

The behaviour of the "individual" agent (cf algorithm 2) builds the list of concessions, then it proposes itself to the rational activity it prefers. When the agent is assigned/unassigned, it informs the "solver" agent. For an unassignment, the "activity" agent waits for a confirmation before sending a new proposal such that a matching is not prematurely returned by the "solver" agent. If an "individual" agent becomes free, it concedes, i.e. sends a proposal to the next rational activity in the list of concessions, possibly until to be definitively assigned to the void activity.

The behavior of the "activity" agent is described by the deterministic finite state automaton in Figure 2. When a proposal is received, it is accepted if the current group is not full (in the state Available). Otherwise, the agent handles the proposals one by one (in the state Casting) by identifying the subgroup which maximizes the egalitarian welfare. Since

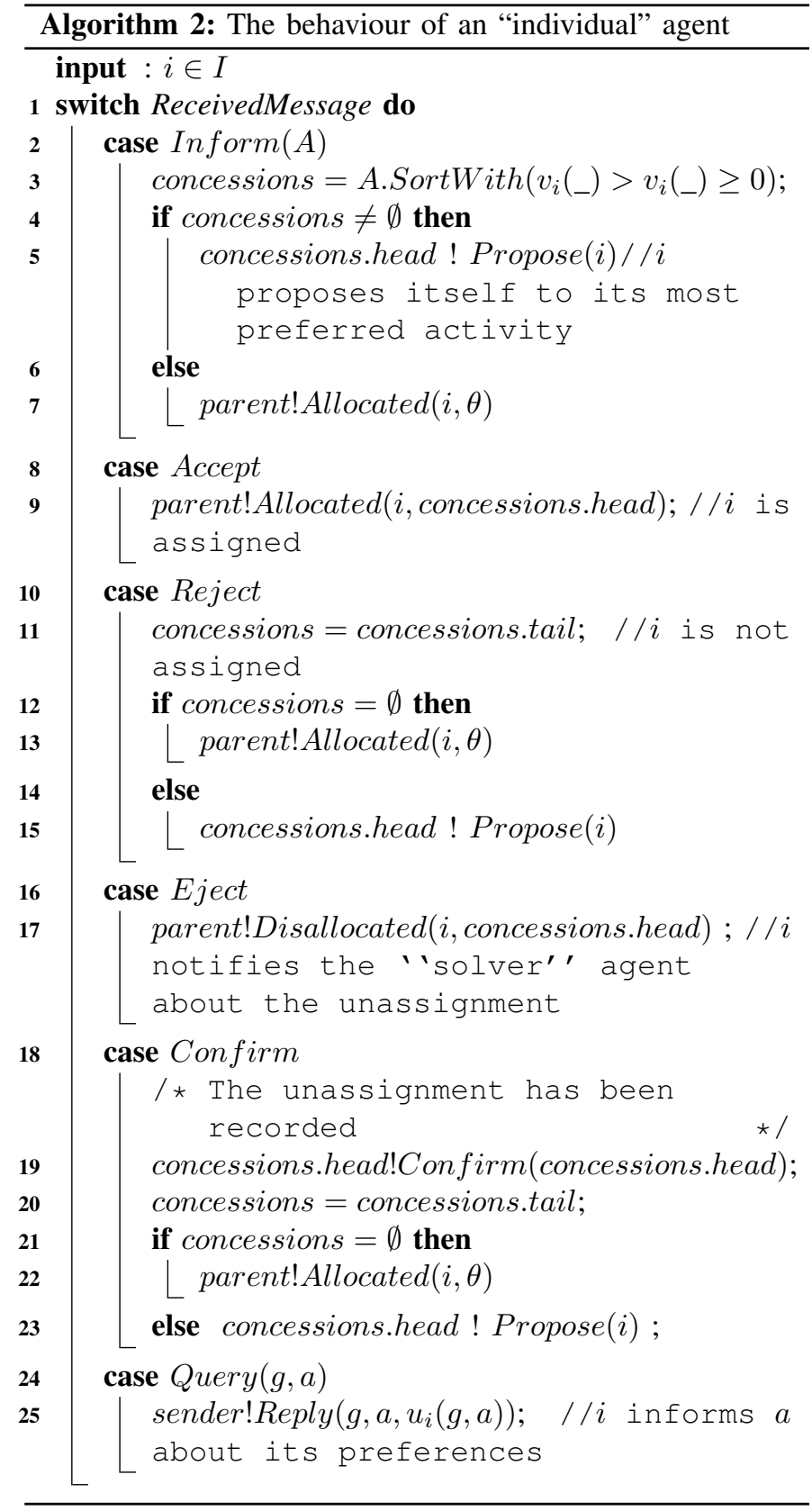

the capacity is reached, the subgroups of size $c_{a}$ or less are considered. If the proposer is not in the best subgroup then the proposal is rejected. Otherwise, the proposal is accepted when the fired members have confirmed the unassignment (in the state Firing). When the proposal is processed, the "activity" agent is ready to evaluate the next proposals, possibly those which have been stashed.

\section{EXPERIMENTS}

Our experiments aim at evaluating the matching reached by our algorithm and the speedup due to its distribution.

We have implemented our prototype with the Scala progamming language and the Akka toolkit [15]. The latter, which is based on the actor model [1], allows us to fill the gap between 


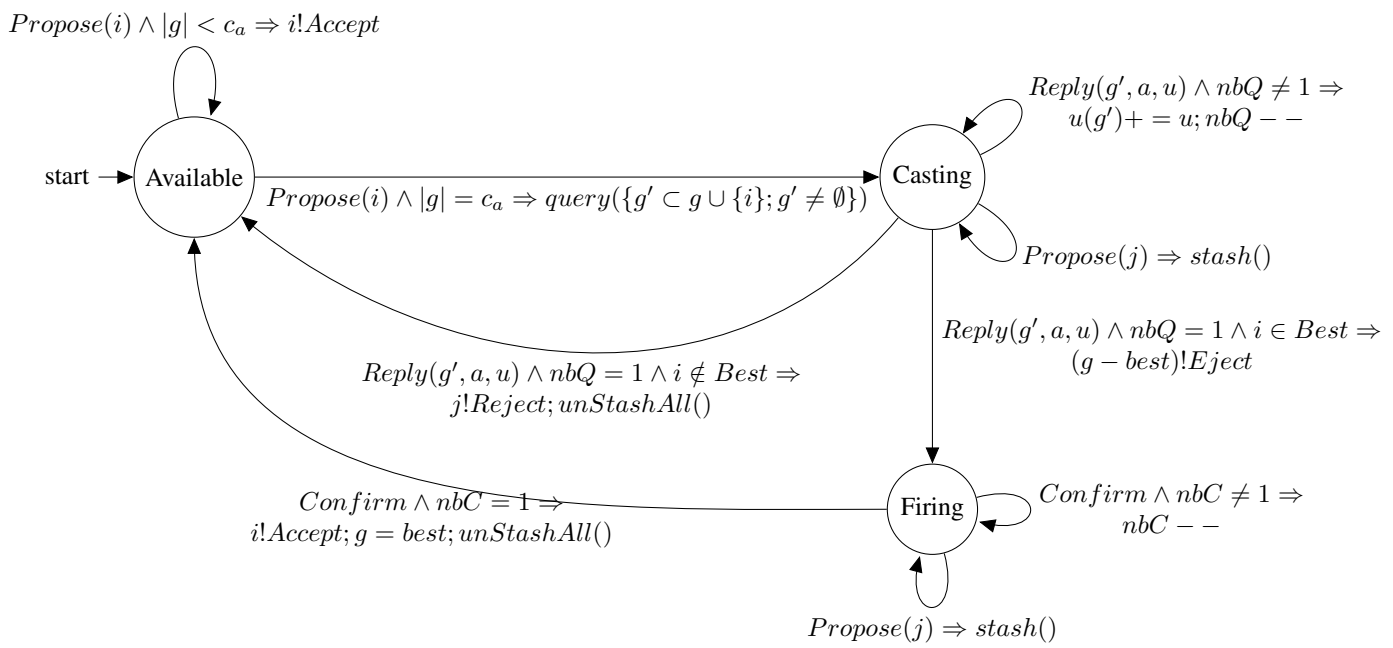

Fig. 2: The behaviour of an "activity" agent

the specification and its implementation. In order to tackle a large number of individuals, we considers the approximation algorithm ${ }^{2}$.

In our experiments, we consider some (pseudo)-randomly generated $A S I A$ instances with $n$ activities and $m$ individuals. Since we aim at evaluating the egalitarian welfare of the outcome of our algorithm, we warrant that the individuals and the activities are strictly rational for all the individuals $\left(\forall i, j \in I \forall a \in A w_{i}(j)>0 \wedge v_{i}(a)>0\right)$ and that all the individuals can be assigned to an activity $\left(\Sigma_{a \in A} c_{a} \geq m\right)$ since it is the case of our practical application. For sake of simplicity, all the activities have the same capacity $(c=\lceil m / n\rceil)$. For each set of parameters $(n$ and $m$ ), we generate 100 instances.

We have implemented a local search algorithm [16] to be compared with our algorithm. This hill-climbing algorithm, which starts with a sound and random matching (where all the individuals are assigned to an activity) and iteratively tries to improve the egalitarian welfare. Two matchings are neighbours if they are identical with an exception for one individual which moves to another activity. If this new activity is full, then all the swaps of individuals with the members of that activity are considered.

Firstly, we compare the egalitarian welfare of the matching reached by our algorithm with the one reached by local search. Figure 3a presents the mean egalitarian welfare obtained for each set of parameters (with $2 \leq n \leq 10$ and $2 \times n \leq m \leq$ 100). Our algorithm overreaches the local search. Indeed, the egalitarian welfare for a $A S I A$ problem is a function with many local optima.

Secondly, we compare the runtime of the centralized version of our approximation algorithm with the decentralized one. Figure $3 \mathrm{~b}$ shows the mean runtimes for each set of parameters (with $2 \leq n \leq 10$ and $2 \times n \leq m \leq 400$ ). While the centralized algorithm is faster when the number of individuals

\footnotetext{
${ }^{2}$ The experiments have been performed with an Intel (R) Core (TM) i7 2.8GHz MacBook Pro with 8 cores and 16GB of RAM.
}

is low $(\sim 40)$, its runtime quickly grows with the number of individuals ( $13 \mathrm{~ms}$ for 100 individuals and 10 activities) while the runtime of the distributed version is less $(9 \mathrm{~ms}$ in the latter case). Moreover, the runtime of the hill-climbing algorithm is too high to be represented in Figure $3 \mathrm{~b}(2,969$ ms for 100 individuals and 10 activities). We can expect a higher runtime if we adopt a local search method such as the simulated annealing without any warranty about the optimality of the outcome.

In summary, the outcome reached by our algorithm seems to be good. Moreover, the distribution of our algorithm allows to speedup (up to 5 times) its runtime.

\section{CONCLUSIONS}

We have presented here the generic problem of individuals/activities where some individuals must be assigned to the activities they enjoy with their favorite partners. Even if the $\mathrm{Nash} / \mathrm{strict}$ core stability is a desirable property, there exists no necessarily such a solution. By contrast, the Pareto-optimality seems not to be discriminative. This is the reason why we have adopted additively separable preferences which allow the evaluation of matchings with the help of the egalitarian welfare. Moreover, this representation of preferences is linear with respect to the number of individuals and it reduces the users' effort for the individual preference elicitation. In order to maximize the egalitarian welfare, we have proposed an inclusive heuristic where the individuals propose themselves to the activities they prefer and eventually concede. The individuals which penalize the egalitarian welfare of a full activity are unassigned and they concede. We have shown that this algorithm always returns a sound matching. By adopting the actor model, we have distributed this algorithm. The difficulty lies in: (i) the detection of the halting (as messages can arrive in a different order from the sending), an "activity" agent must wait for the confirmation of an unassignment before assigning a new individual); (ii) the synchronization (as the acceptance of an individual depends on the group, an 

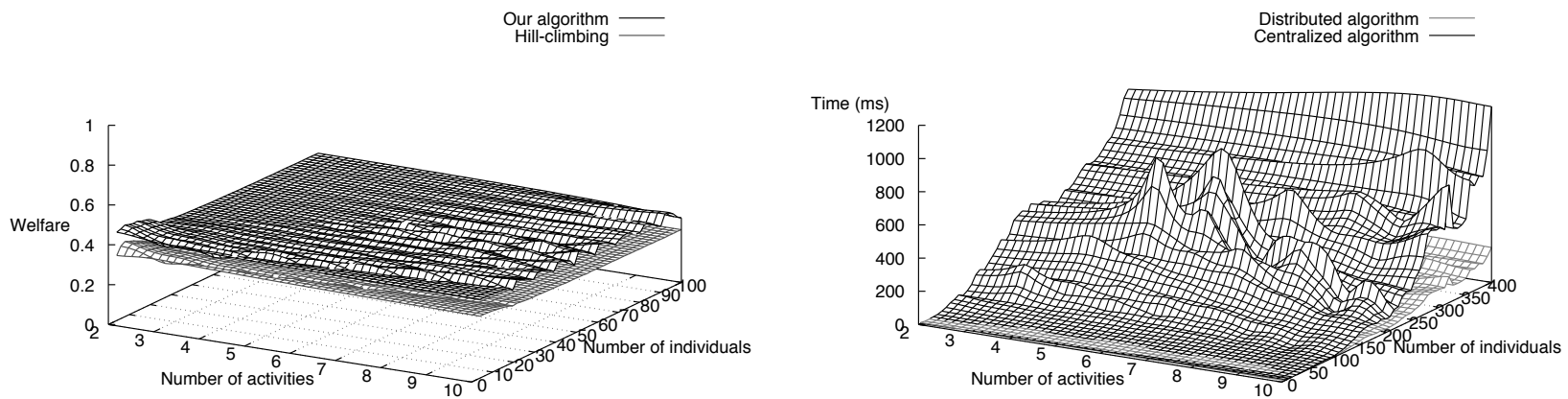

Fig. 3: Egalitarian welfare of matching reached by our algorithm/hill-climbing (at left) and decentralized/centralized algorithm runtime (at right)

"activity" agent must deal with the proposals one-by-one). Our experiments show that our algorithm benefits of the structural properties of two-sided matching and it overreaches local search techniques. Finally, the distribution of our algorithm speeds up its execution (up to 5 times).

In future works, we aim at evaluating our matching engine with the real-world data from our project.

\section{ACKNOWLEDGMENT}

This work is part of the PartENS research project supported by the Nord-Pas de Calais region (researcher/citizen research projects). We thank the reviewers for their useful comments.

\section{REFERENCES}

[1] W. D. Clinger, "Foundations of actor semantics," Ph.D. dissertation, Massachusetts Institute of Technology, 1981.

[2] J. Dreze and J. Greenberg, "Hedonic coalitions: Optimality and stability," Econometrica, vol. 48, pp. 987-1003, 1980.

[3] H. Aziz, F. Brandt, and H. G. Seedig, "Computing desirable partitions in additively separable hedonic games," AIJ, vol. 195, pp. 316-334, 2013.

[4] C. Ballester, "NP-completeness in hedonic games," $G E B$, vol. 49, no. 1, pp. 1-30, 2004.

[5] T. C. Schelling, The strategy of conflict. Harvard university press, 1980.
[6] A. Darmann, E. Elkind, S. Kurz, J. Lang, J. Schauer, and G. Woeginger, "Group activity selection problem," in Proceedings of the 8th International Conference on Internet and Network Economics. Liverpool, UK: Springer Berlin Heidelberg, 2012, pp. 156-169.

[7] A. Igarashi, D. Peters, and E. Elkind, "Group activity selection on social networks," in Proc. of AAAI, 2017, pp. 565-571.

[8] D. Gale and L. S. Shapley, "College admissions and the stability of marriage," The American Mathematical Monthly, vol. 69, pp. 9-14, 1962.

[9] D. F. Manlove, Algorithmics of Matching Under Preferences. World Scientific, 2014.

[10] P. Everaere, M. Morge, and G. Picard, "Minimal Concession Strategy for Reaching Fair, Optimal and Stable Marriages," in Proc. of AAMAS, 2013, pp. 1319-1320.

[11] A. Nongaillard and S. Picault, "Multilevel agent-based modelling for assignment or matching problems," in Proc. of ECAI, 2016, pp. 15611562.

[12] C. Boutilier, I. Caragiannis, S. Haber, T. Lu, A. D. Procaccia, and O. Sheffet, "Optimal social choice functions: A utilitarian view," AIJ, vol. 227, pp. 190-213, 2015.

[13] A. K. Sen, Collective Choice and Social Welfare. North-Holland, 1970.

[14] H. Moulin, Fair Division and Collective Welfare. The MIT Press, 2002.

[15] "The ScaIA library," (Date last accessed 15-September-2017). [Online]. Available: https://github.com/maximemorge/ScaIA

[16] S. Russell and P. Norvig, Artificial Intelligence: A Modern Approach. Pearson Education, 2003, ch. Informed Search and Exploration, pp. 94136, 2nd edition. 